[Radiocarbon, Vol. 16, No. 3, 1974, P. 331-357]

\title{
RIKEN NATURAL RADIOCARBON MEASUREMENTS VIII
}

FUMIO YAMASAKI, CHIKAKO HAMADA, and TATSUJI HAMADA

Institute of Physical and Chemical Research (RIKEN)

Wako-shi, Saitama Pref, Japan 351

The ${ }^{14} \mathrm{C}$ dates given below are continued from our previous list ( $R, 1972, \mathrm{v} 14, \mathrm{p}$ 223-238), and results obtained mainly during 1971-2 are described. A $2.7 \mathrm{~L}$ stainless steel counter and a $3.3 \mathrm{~L}$ copper counter are used as previously, yielding background counting rates of 6.9 and $6.0 \mathrm{cpm}$, respectively, when filled with dead $\mathrm{CO}_{2}$ at ca $1.8 \mathrm{~atm}$. Dates have been calculated on the basis of the ${ }^{14} \mathrm{C}$ half-life of $5568 \mathrm{yr}$ and $95 \%$ of NBS oxalic acid is modern standard. No correction has been made for any of the samples in this list.

\section{SAMPLE DESCRIPTIONS}

I. GEOLOGIC SAMPLES

\section{Tokyo Bay series}

$$
\text { A. Japan }
$$

Samples from boring cores. Coll 1970 by K Kojima; subm by K Kojima and K Kuwahara, Public Works Res Inst.

N-1296. Off Kawasaki (28-H-14')

Shell from coarse sand, elev $-91.4 \mathrm{~m}$ TP, off Kawasaki $\left(35^{\circ} 27^{\prime} \mathrm{N}\right.$, $\left.139^{\circ} 54^{\prime} \mathrm{E}\right)$, Borehole 28, water depth $13.7 \mathrm{~m}$ TP.

\section{N-1297. Ukishima-cho (32-H-11')}

$26,100 \pm 860$

Shell from silty fine sand, elev $-85.8 \mathrm{~m}$ TP, at Ukishima-cho, Kawasaki city $\left(35^{\circ} 32^{\prime} \mathrm{N}, 139^{\circ} 47^{\prime} \mathrm{E}\right)$, Borehole 32 , surface elev $2.8 \mathrm{~m}$ TP.

N-1298. Ukishima-cho (32-H-1)

$385 \pm 85$

Wood from alluvial sandy silt (N-value $2 \sim 4)$, elev $-11.3 \mathrm{~m}$ TP, at same borehole as $\mathrm{N}-1297$. Comment $(\mathrm{KK})$ : comparing with some ${ }^{14} \mathrm{C}$ dates and geologic data (Kanto Regional Construction Bureau, 1973), date of N-1926 is reasonable but N-1297 seems rather young. Borehole 32 is on reclaimed land.

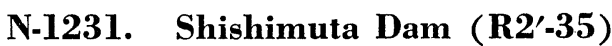

$>\mathbf{3 7 , 8 0 0}$

Charcoal from buried talus overlain by Aso lava, from an adit dug for dam foundation survey at river cliff of Kusu R, a branch of Chikugo

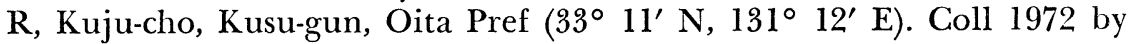
$\mathrm{K}$ Hayashi, Chikugogawa Sta, Ministry of Construction; subm by $\mathrm{K}$ Kojima and K Kuwahara. Comment (KK): Aso lava is correlated to Young Aso lava dated ca 30,000 вP in other place (Ariake Bay Res Group, 1965).

\section{Yahagi River series}

Samples from boring core taken from alluvium of Yahagi R, Ugaike- 
cho, Nishio-shi, Aichi Pref (34 50' N, $137^{\circ} 5^{\prime}$ E). Coll 1971 and subm by A Moriyama, Aichi Univ Education.

\section{N-1262. Yahagi River 1}

$8850 \pm 145$

Peat from muddy sand ca $28 \mathrm{~m}$ below surface.

6900 BC

N-1263. Yahagi River 2

$4180 \pm 95$

$2230 \mathrm{BC}$

Peat from sand ca $7 \mathrm{~m}$ below surface, near base of upper sand overlying mud.

\section{N-1264. Yahagi River 3}

$3250 \pm 95$ 1300 BC

Black organic soil from sand as above, ca $3 \mathrm{~m}$ below surface. Comment (AM): sedimentary structure of alluvia in drainage basin of Yahagi $\mathrm{R}$ at coastal area is well stratified and slaty. Structure at inland area, however, $\mathrm{N}$ of New Tokaido Line, is disordered and confused. Evidently, they depend upon expanse of transported bed loads by tidal or off-shore currents in open sea and lens-like deposition of coarse materials in channel belts on alluvial upland or in closed sea. Using measured ${ }^{14} \mathrm{C}$ age of these layers at coastal area, lower sands accumulated from ca 10,000 вP, middle muds са 7000 вP, and upper sands са 4500 вр The coast of the maximum "Jomon" transgression may have lain a little $\mathrm{N}$ of the New Tokaido Line. By rapid and successive alluviations, the coast at the age of late "Yayoi" may have lain near the line which links Isshiki with Kira (Moriyama and Ozawa, 1972).

\section{B. Great Britain}

\section{N-962. Gate Helmsley (SE65/8463)}

$6030 \pm 140$

Wood from 2 to $2.5 \mathrm{~m}$ beneath eolian sand, inner side of York Moraine near Gate Helmsley, Yorkshire $\left(53^{\circ} 58^{\prime} \mathrm{N}, 0^{\circ} 58^{\prime} \mathrm{W}\right)$. Coll and subm 1970 by B Matthews, Soil Survey England \& Wales. Comment $(\mathrm{BM})$ : date indicates area was wooded during Atlantic Period; later, wind blown sand accumulated in lee of moraine, probably after Neolithic forest clearance.

\section{East Moor series}

Samples from various depths at East Moor, Sutton-on-the-Forest, Yorkshire $\left(54^{\circ} 4^{\prime} \mathrm{N}, 1^{\circ} 4^{\prime} \mathrm{W}\right)$. Coll and subm 1970 by B Matthews.

N-963. East Moor 1 (SE66/1041/1)

Sandy peat from 118 to $121 \mathrm{~cm}$.

\section{N-964. East Moor 2 (SE66/1041/2)}

Sandy peat from 122.5 to $124 \mathrm{~cm}$.

\section{$11,000 \pm 200$ 9050 BC}

$11,200 \pm 160$ 9250 BC

General Comment (BM): those and N-488 (10,700 $\pm 190, \mathrm{R}, 1969$, v 11, p 455), and N-820 (9950 $\pm 180, \mathrm{R}, 1972, \mathrm{v} 14, \mathrm{p} 227)$ limit dates for 
deposition of eolian sand in Vale of York and for Aller $\phi \mathrm{d}$ interstadial in the area (ie, 9950 to $11,200 \mathrm{yrs} \mathbf{B P}$ ). Evidence suggests Aller $\phi \mathrm{d}$ started later in Yorkshire than in S England and lasted till a later date (Matthews, 1970; 1971).

\section{N-965. East Moor 3 (SE66/1041/3)}

$6400 \pm 310$

Plant roots embedded in calcareous clayey till from depth 138 to $180 \mathrm{~cm}$. Comment $(\mathrm{BM})$ : sample either contaminated or roots are from vegetation of Atlantic period.

\section{PEDOLOGIC SAMPLES}

Total organic carbon, unless otherwise stated, of samples from humic horizon in volcanic ash and muck from various localities, coll 1971 to 1972 by Y Yamada, Natl Inst Agric Sci, are dated to determine relationship between soil age and properties of humus in soil.

\section{Kitamoto series}

Samples from various depths in volcanic ash soils developed at Kitamoto, Saitama Pref.

\section{N-850. Kitamoto 1-1}

$$
1120 \pm 110
$$

From depth 0 to $45 \mathrm{~cm}, A_{p}$ horizon, at Yamanaka, Kitamoto-cho, Kitaadachi-gun, Saitama Pref $\left(36^{\circ} 2^{\prime} \mathrm{N}, 139^{\circ} 33^{\prime} \mathrm{E}\right)$. Carbon content: $2.63 \%$.

\section{N-849. Kitamoto 1 -2}

$$
1540 \pm 110
$$

From depth 45 to $50 \mathrm{~cm}$, IIA horizon. Carbon content: $3.24 \%$.

\section{N.957. Kitamoto 11 -2}

From depth 28 to $60 \mathrm{~cm}$, IIA horizon on Omiya plateau at Miyauchi, Kitamoto-cho $\left(36^{\circ} 2^{\prime} \mathrm{N}, 139^{\circ} 32^{\prime} \mathrm{E}\right)$. Carbon content: $5.20 \%$. Comment: (YY): horizon yielding N-957 corresponds to that of N-849. However, $\mathrm{N}-957$ was expected to be older from character of humus.

\section{N.958. Kitamoto $13-3$}

$18,800 \pm 370$

From depth 160 to $180 \mathrm{~cm}$, at Haramamuro, Konosu city $\left(36^{\circ} 3^{\prime} \mathrm{N}\right.$, $139^{\circ} 31^{\prime} \mathrm{E}$ ). Horizon yielding N-958 is considered to overlie Lower Tachikawa Loam bed. Carbon content: $4.41 \%$.

\section{Fukui series} environs.

Sample from various depths in organic soils in Fukui city and its

N-1082. Fukui 1-2, soil organic matter

$\mathrm{M}_{1}$ horizon of peaty soil, depth 20 to $40 \mathrm{~cm}$, at Mitome, Shimizu-cho, Nyu-gun, Fukui Pref $\left(36^{\circ} 1^{\prime} \mathrm{N}, 136^{\circ} 9^{\prime}\right.$ E). Carbon content: $2.81 \%$. 
N-1077. Fukui 1-2, $\mathrm{FeCO}_{3}$

Modern

$\mathrm{FeCO}_{3}$ concretion, same horizon as above.

N-1083. Fukui 1-4, soil organic matter

$1270 \pm 100$

$\mathrm{M}_{2}$ horizon, depth 40 to $50 \mathrm{~cm}$. Carbon content: $9.05 \%$.

N-1084. Fukui 2-2, soil organic matter

$1330 \pm 110$

M horizon of muck soil, depth 20 to $40 \mathrm{~cm}$, at Ryo-machi, Fukui city $\left(36^{\circ} 4^{\prime} \mathrm{N}, 136^{\circ} 16^{\prime} \mathrm{E}\right)$. Carbon content: $2.89 \%$.

N-1078. Fukui 2-2, $\mathrm{FeCO}_{3}$

$\mathrm{FeCO}_{3}$ concretion, same horizon as above.

AD 1055

$895 \pm 100$

N-1085. Fukui 3-2, soil organic matter

M horizon of muck soil, depth 16 to $35 \mathrm{~cm}$, at Sakai-cho, Sakai-gun, Fukui Pref $\left(36^{\circ} 9^{\prime} \mathrm{N}, 136^{\circ} 13^{\prime} \mathrm{E}\right)$. Carbon content: $4.43 \%$.

N-1079. Fukui 3-2, $\mathrm{FeCO}_{3}$

$\mathrm{FeCO}_{3}$ concretion, same horizon as above.

Modern

N-1206. Fukui 26-2

M horizon of muck soil, depth 30 to $50 \mathrm{~cm}$, at Hamajima, Kawanishicho, Fukui city $\left(36^{\circ} 9^{\prime} \mathrm{N}, 136^{\circ} 7^{\prime} \mathrm{E}\right)$. Carbon content: $4.46 \%$.

\section{N-1207. Fukui 27-4}

$1710 \pm 90$

M horizon of muck soil, depth 33 to $44 \mathrm{~cm}$, at Tameyori, Kawanishicho, Fukui city $\left(36^{\circ} 8^{\prime} \mathrm{N}, 136^{\circ} 7^{\prime} \mathrm{E}\right)$. Carbon content: $14.24 \%$.

N-1208. Fukui 28-4

$2530 \pm 120$

M horizon of muck soil, depth 37 to $60 \mathrm{~cm}$, at Yawata, Kawanishicho, Fukui city $\left(36^{\circ} 9^{\prime} \mathrm{N}, 136^{\circ} 8^{\prime} \mathrm{E}\right)$. Carbon content: $6.13 \%$.

N-1209. Fukui 29-1

Modern

$A_{p}$ horizon of peat soil, depth 0 to $11 \mathrm{~cm}$, at Kinoshita, Kawanishi-cho, Fukui city $\left(36^{\circ} 8^{\prime} \mathrm{N}, 136^{\circ} 8^{\prime} \mathrm{E}\right)$. Carbon content: $4.10 \%$.

N-953. Fukui 29-2 (1)

$$
1480 \pm 110
$$

Peat from depth 21 to $40 \mathrm{~cm}$ of $\mathrm{P}$ horizon. No pretreatment was made.

N-954. Fukui 29-2 (2)

$1470 \pm 110$

Above sample was washed with $0.5 \% \mathrm{NaOH}$, air-dried, and dated. 
N-955. Fukui 29.2 (3)

$1460 \pm 140$

Humic acid extracted from N-953.

AD 490

\section{Imaichi series}

Sample from various depths in volcanic ash soils in Imaichi city and its environs.

\section{N-1180. Imaichi 3-1}

$1370 \pm 100$

$\mathrm{A}_{11}$ horizon from depth 0 to $20 \mathrm{~cm}$ at $\mathrm{MD} 50$ N, $139^{\circ} 43^{\prime}$ E). Carbon content: $19.7 \%$.

\section{N-1181. Imaichi 3-2}

$3130 \pm 110$

$\mathrm{A}_{12}$ horizon from depth 20 to $35 \mathrm{~cm}$ Carbon $1180 \mathrm{BC}$ Carbon content: $18.7 \%$. Comand GaK-1328 (R, 1969, v 11, p 300).

\section{N-1182. Imaichi 3-3}

$A_{13}$ horizon from depth 35 to $50 \mathrm{~cm}$. Carbon content: $17.6 \%$.

$$
4140 \pm 110
$$

2190 BC

\section{N-1183. Imaichi 3-4}

$5560 \pm 125$

$\mathrm{A}_{14}$ horizon from depth 50 to $70 \mathrm{~cm}$. Carbon content: $12.0 \%$.

\section{N-956. Imaichi 3-5}

$5360 \pm 120$

$\mathrm{A}_{3}$ horizon from depth 70 to $84 \mathrm{~cm}$ underlain by Shichihonzakura pumice layer. Carbon content: $6.78 \%$.

\section{N-1184. Imaichi 4-1}

$$
1690 \pm 100
$$

A horizon from depth 0 to $28 \mathrm{~cm}$, at Osawa, Imaichi city $\left(36^{\circ} 42^{\prime} \mathrm{N}\right.$, $139^{\circ} 45^{\prime} \mathrm{E}$ ). Imaichi 4 soil is used as upland field. Carbon content: $14.9 \%$.

N-1185. Imaichi 4-2

$4290 \pm 120$

$\mathrm{A}_{12}$ horizon from depth 28 to $46 \mathrm{~cm}$. Carbon content: $12.1 \%$.

\section{N-1186. Imaichi 4-3}

$$
5900 \pm 125
$$

$\mathrm{A}_{13}$ horizon from depth 46 to $60 \mathrm{~cm}$. Carbon content: $9.0 \%$.

\section{N-1418. Imaichi 8-1}

$$
\begin{array}{r}
1010 \\
\text { AD } 940
\end{array}
$$

From depth 0 to $15 \mathrm{~cm}, A_{p}$ horizon, at Yokaichi, Imaichi city $\left(36^{\circ}\right.$ $42^{\prime} \mathrm{N}, 139^{\circ} 46^{\prime} \mathrm{E}$ ). Profile characteristics are nearly equal to Imaichi 4 soil. Used as paddy field. Carbon content: $9.21 \%$ 
N-1187. Imaichi 5-1

$$
670 \pm 100
$$

AD 1280

$A_{p}$ horizon from depth 0 to $15 \mathrm{~cm}$, at Yokaichi, Imaichi city $\left(36^{\circ} 42^{\prime}\right.$ N, $139^{\circ} 46^{\prime}$ E). Carbon content: $13.9 \%$.

N.1188. Imaichi 5-2

$$
1820 \pm 100
$$

$A_{p g}$ horizon from depth 15 to $22 \mathrm{~cm}$. Carbon content: $11.8 \%$.

N-1189. Imaichi 5-3

$$
2060 \pm 90
$$

$110 \mathrm{BC}$

$A_{12}$ horizon from depth 22 to $37 \mathrm{~cm}$. Carbon content: $12.3 \%$.

\section{N-1190. Imaichi 5-4}

$$
2980 \pm 110
$$

1030 BC

$\mathrm{A}_{13}$ horizon from depth 37 to $61 \mathrm{~cm}$. Carbon content: $12.2 \%$.

\section{N-1360. Imaichi 5-5}

$A_{3}$ horizon from depth 61 to $67 \mathrm{~cm}$. Carbon content: $7.60 \%$. Comment (YY): considered to roughly correspond to Imaichi $3-4$ and $3-5$ (N-1183, N-950, above).

\section{N-1191. Imaichi 6-1}

$$
415 \pm 100
$$

$A_{p}$ horizon from depth 0 to $18 \mathrm{~cm}$ of volcanic ash soil derived from secondary deposits of volcanic ash on narrow valley plain on middle terrace, at Yokaichi, Imaichi city $\left(36^{\circ} 42^{\prime} \mathrm{N}, 139^{\circ} 45^{\prime} \mathrm{E}\right)$. Imaichi 6 soil is used as paddy field. Carbon content: $9.7 \%$.

\section{N-1192. Imaichi 6-3}

$$
1790 \pm 100
$$

$$
\text { AD } 160
$$

$\mathrm{A}_{12}$ horizon from depth 22 to $35 \mathrm{~cm}$. Carbon content: $9.4 \%$.

\section{N-1193. Imaichi 6-4}

$$
1800 \pm 160
$$

$\mathrm{A}_{13}$ horizon from depth 35 to $53 \mathrm{~cm}$. Carbon content: $5.4 \%$.

N-1194. Imaichi 7-1

$$
\text { AD } 1785
$$

$A_{p}$ horizon from depth 0 to $14 \mathrm{~cm}$, at Shionomuro, Imaichi city $\left(36^{\circ} 44^{\prime} \mathrm{N}, 139^{\circ} 48^{\prime} \mathrm{E}\right)$. Carbon content: $8.4 \%$.

\section{N-1195. Imaichi 7-2}

$\mathrm{A}_{12}$ horizon from depth 14 to $26 \mathrm{~cm}$. Carbon content: $9.7 \%$.

N-1196. Imaichi 7-3

$\mathrm{A}_{13}$ horizon from depth 26 to $60 \mathrm{~cm}$. Carbon content: $11.5 \%$. 


\title{
N-1361. Imaichi 7-4
} $9.78 \%$.

A therizon from depth 60 to $80 \mathrm{~cm}$ (Aodo

\section{Tokorozawa series}

Sample from various depths in volcanic ash beds in Tokorozawa city and its environs.

\section{N-1382. Tokorazawa 12-1}

$A_{p}$ horizon from depth 0 to $22 \mathrm{~cm}$, at Arahata, Tokorozawa city $\left(35^{\circ} 46^{\prime} \mathrm{N}, 139^{\circ} 27^{\prime} \mathrm{E}\right)$. Carbon content: $2.22 \%$.

N-1383. Tokorazawa 12 -2

$$
2830 \pm 100
$$

A horizon from depth 22 880 BC

\author{
880 B.
}

N-1384. Tokorazawa 17-1

$630 \pm 70$

$A_{p}$ horizon from depth 0 to $25 \mathrm{~cm}$, at Kamiyamaguch city $\left(35^{\circ} 46^{\prime} \mathrm{N}, 139^{\circ} 25^{\prime} \mathrm{E}\right)$ to $25 \mathrm{~cm}$, at Kamiyamaguchi, Tokorozawa Asamizo series

Sample from various depths of humus horizons in volcanic ash soil at Asamizo-dai, Sagamihara city, Kanagawa Pref ( $\left.35^{\circ} 46^{\prime} \mathrm{N}, 139^{\circ} 25^{\prime} \mathrm{E}\right)$.

\section{N-1419. Asamizo 1}

$$
800 \pm 80
$$

$\mathrm{A}_{\mathrm{p}}$ horizon from depth 0 to $21 \mathrm{~cm}$. Carbon content: $8.36 \%$.

\section{N-1420. Asamizo 2}

$1680 \pm 80$

II $\mathrm{A}_{11}$ horizon from depth 21 to $43 \mathrm{~cm}$. Carbon content: $7.95 \%$.

N-1421. Asamizo 3

$$
3160 \pm 90
$$

II $\mathrm{A}_{12}$ horizon from depth 43 to $50 \mathrm{~cm}$. Carbon content: $6.14 \%$.

\section{N-1422. Asamizo 4}

$4220 \pm 90$

III A horizon from depth 50 to

N-1423. Asamizo 5

IV A horizon from depth $80 \mathrm{~cm}$. Carbon content: $7.30 \%$.

$$
6500 \pm 120
$$

4550 BC

N-1424. Asamizo 6

$7060 \pm 130$

5110 BC

VB horizon from depth 130 to $150 \mathrm{~cm}$. Carbon content: $2.64 \%$. 


\section{A. Japan}

\section{Sanrizuka series}

Charcoal of coniferous tree from ca $1.2 \mathrm{~m}$ below ground surface at construction site of New Tokyo International Airport at Kogome, Narita city, Chiba Pref $\left(35^{\circ} 46^{\prime} \mathrm{N}, 140^{\circ} 24^{\prime} \mathrm{E}\right)$. Lens shaped charcoal concentration, $120 \mathrm{~cm}$ in diam and ca $30 \mathrm{~cm}$ thick, from lower part of Level 5, considered Tachikawa Loam. Same horizon yielded stone tools such as knife, blade, hand axe, etc (Furuuchi, 1971). Coll 1971 by C Watanabe; subm by G Nishino, Hokuso Kosha, Chiba Pref.

\section{N-1080. Sanrizuka 1}

Charcoal from A55,402,002.

\section{N-1081. Sanrizuka 2}

Charcoal from A55,402,007.
$29,300 \pm 980$

\section{$27,350 \mathrm{BC}$}

$28,700 \pm 920$

26,750 BC

\section{Hamabekkai series}

Material from archaeol remains at Hamabekkai, Bekkai-cho, Notsuke-gun, Hokkaido $\left(43^{\circ} 27^{\prime} \mathrm{N}, 144^{\circ} 37^{\prime} \mathrm{E}\right)$. Coll and subm 1971 by T Iwasaki, Tokyo Univ Education.

\section{N-1111. Hamabekkai 1}

$1130 \pm 110$

Charred timber from probably burned house remnant (H-4) of Post Jomon period.

\section{N-1112. Hamabekkai 2}

$895 \pm 110$

Burned wood from floor of house remnant of Okhotsk culture period.

N-1113. Hamabekkai 3

$$
1020 \pm 100
$$

AD 930

Charcoal from hearth of dwelling pit (H-10) of Latest Satsumon period. Probably assoc with coin of Ming dynasty. Comment (TI): other dates of remains of same period are: GaK-186 and -187 (R, 1963, v 5, p 116) and TK-4, -17, -52, and -53 (R, 1968, v 10, p 147; R, 1969, v 11, p 512) and I-555.

\section{N-1114. Hamabekkai 4}

Burned wood from floor of house remnant (H-20) of Middle Jomon period.

\section{Suwanohara series}

Material from floor of burned houses of Latest Yayoi or Early Kofun period at Suwanohara, Matsudo city, Chiba Pref $\left(35^{\circ} 47^{\prime} \mathrm{N}\right.$, $\left.139^{\circ} 54^{\prime} \mathrm{E}\right)$. Coll and subm by T Iwasaki. 


\section{N-1115. Suwanohara 1}

$1930 \pm 100$

Charcoal from S-1.

N-1116. Suwanohara 2

$1820 \pm 100$

Charcoal from S2.

N-1117. Suwanohara 3

Charcoal from S-22.

$1830 \pm 100$

AD 120

General Comment (TI): dates older than expected by ca $200 \mathrm{yr}$.

\section{Kode series}

Material from archaeol remain at Kode, Matsudo city, Chiba Pref $\left(35^{\circ} 47^{\prime} \mathrm{N}, 139^{\circ} 54^{\prime} \mathrm{E}\right)$. Coll by I Yawata; subm 1971 by T Iwasaki.

\section{N-1156. Kode 1}

$5790 \pm 140$

Charcoal from shell mound, assoc with pottery of $\mathbf{3 8 4 0 ~ B C}$ type of Early Jomon period.

\section{N-1157. Kode 2}

$5900 \pm 115$ 3950 BC

Charcoal from floor of Dwelling Pit 202, assoc with pottery of Sekiyama type of Early Jomon period.

\section{Kainohana series}

Material from Kainohana shell mound, Hachigasaki, Matsudo city, Chiba Pref $\left(35^{\circ} 49^{\prime} \mathrm{N}, 139^{\circ} 56^{\prime} \mathrm{E}\right)$. Coll by I Yawata; subm 1971 by T Iwasaki.

\section{N-1429. Kainohana 1}

$3940 \pm 105$

Charcoal from dwelling pit, assoc with pottery of Horinouchi I type of Late Jomon period.

\section{N-1430. Kainohana 2}

$3840 \pm 190$

Charcoal from shell bed, assoc with pottery of Kasori BI type of Late Jomon period.

\section{N.1431. Kainohana 3}

$4170 \pm 105$

Charcoal from shell bed, assoc with pottery of Kasori $\mathrm{E}$ type of Middle Jomon period.

\section{N-1259. Kotani}

$$
3470 \pm 85
$$

Wood fragment from archaol remains at $\mathrm{Kotani}$ Kasai city $\mathbf{1 5 2 0}$ Pref $\left(34^{\circ} 53^{\prime} \mathrm{N}, 134^{\circ} 52^{\prime} \mathrm{C}\right)$ Coll 1971 by Pref $\left(34^{\circ} 53^{\prime} \mathrm{N}, 134^{\circ} 52^{\prime} \mathrm{E}\right)$. Coll 1971 by Y Maeda; subm by K Huzita. Comment $(\mathrm{KH})$ : assoc artifacts suggest 4 th or 5 th century occupation. 


\section{Tripod Cinerary Urn}

Human bone and charcoal contained in a cinerary urn supported with 3 legs of animal-leg shape, owned by Tokuzo-ji monastery, at Higashi-Murayama city, Tokyo $\left(35^{\circ} 46^{\prime} \mathrm{N}, 139^{\circ} 28^{\prime} \mathrm{E}\right)$. Coll by S Asaki; subm 1971 by T Imadate (Asaki, 1957).

\section{N-1212-1. Human bone}

$$
\begin{array}{r}
1120 \pm 165 \\
\text { AD } 830
\end{array}
$$

\section{N-1212-2. Charcoal}

\section{Snyder site series}

\section{B. United States}

Charcoal from Snyder site, $\mathrm{N}$ of El Dorado, Butler Co, Kansas $\left(37^{\circ}\right.$ $\left.52^{\prime} \mathrm{N}, 96^{\circ} 49^{\prime} \mathrm{W}\right)$. Coll 1968 to 1971 and subm 1972 by R Grosser, Univ Kansas. Assoc with Archaic materials except N-1280, for which cultural affiliation has not yet been ascertained. Comment: other dates of this series are found in R, 1972, v 14, p 229-30.

\section{N-1276. Synder site 1}

$2060 \pm 80$

From depth $45 \mathrm{~cm}$, in homogeneous, dark brown, mottled clayey soil. Comment (RG): previous date for 40 to $55 \mathrm{~cm}$ level was $1970 \pm 110$ (N-769).

\section{N-1277. Snyder site 2}

$3240 \pm 85$ hearth at 100 to $125 \mathrm{~cm}$ yielded $3650 \pm 140(\mathrm{~N}-770)$.

\section{N-1278. Synder site 3}

From depth $128 \mathrm{~cm}$, same soil zone as $\mathrm{N}-1276$ and -1277 . Comment (RG): level 125 to $140 \mathrm{~cm}$ from another area of site yielded $3910 \pm 160$ $(\mathrm{N}-771)$.

\section{N-1279. Snyder site 4}

From depth $178 \mathrm{~cm}$, in transition zone between homogeneous dark brown clayey soil and underlying yellowish brown clay.

\section{N-1280. Snyder site 5}

$4600 \pm 125$

From depth $250 \mathrm{~cm}$ in yellowish brown clay, assoc with numerous flakes, grinding stone, and chipped stone. Cultural material absent from preceding $0.5 \mathrm{~m}$.

\section{N-1265. Ponshewaing Point site (3182.54)}

$3030 \pm 95$ 1080 BC

Charcoal from hearth, Ponshewaing Point site, Emmet Co, Michigan $\left(45^{\circ} 25^{\prime} \mathrm{N}, 84^{\circ} 48^{\prime} \mathrm{W}\right)$. Coll 1970 by W A Lovis; subm 1972 by the Museum, Michigan State Univ. 
N-1266. Pine River Channel site (3683.10)

Charcoal from hearth, Pine River Channel site, Charlevoix Co, Michigan $\left(45^{\circ} 19^{\prime} \mathrm{N}, 85^{\circ} 16^{\prime} \mathrm{W}\right)$. Coll 1971 by C E Cleland; subm 1972 by the Museum, Michigan State Univ.

\section{N-1267. Eagle Island site (3458.7.7)}

$2400 \pm 80$

Charcoal from hearth, Eagle Island site, Charlevoix Co, Michigan $\left(45^{\circ} 18^{\prime} \mathrm{N}, 85^{\circ} 1^{\prime} \mathrm{W}\right.$ ). Coll 1969 by C E Cleland; Michigan State Univ.

N-1268. O'Neill site (3468.15.14)

Charcoal from lower occupation zone, O'Neill site, Charlevoix Co, Michigan $\left(45^{\circ} 36^{\prime} \mathrm{N}, 85^{\circ} 21^{\prime} \mathrm{W}\right)$. Coll 1971 by W A Lovis; subm 1972 by the Museum, Michigan State Univ.

\section{Indian Mound Park series}

Material from cap area of 2 burial mounds containing 21 persons of both primary and secondary interment at Indian Mound Park (20Ibl), Rolland Township, Isabella Co, Michigan $\left(43^{\circ} 31^{\prime} \mathrm{N}, 84^{\circ} 59^{\prime} \mathrm{W}\right)$. Late Woodland ceramics, quartz projectile point and celt were found, assoc with cap layers. Coll 1971 by K C Carstens; subm by Maria Campbell, Central Michigan Univ.

\section{N-1289. Indian Mound Park 1}

$1070 \pm 75$

Charcoal of occurrence 530R515, Level 2. AD 880

N-1290. Indian Mound Park 2

$1080 \pm 75$

Charcoal of occurrence 545R500, Level 2. AD 870

\section{Lilbourn series}

Charcoal from burial on Lilbourn archaeol site, 23NM38, fortified Middle Mississippian townsite in New Madrid Co, SE Missouri $\left(36^{\circ} 34^{\prime}\right.$ $\left.\mathrm{N}, 89^{\circ} 36^{\prime} \mathrm{W}\right)$. Coll and subm by $\mathrm{A} \mathrm{H}$ Chapman, Univ MissouriColumbia.

\section{N-1232. Lilbourn 1}

Cat No. 71-1884.

\section{N-1233. Lilbourn 2}

Cat No. 71-1885.

$$
\begin{array}{r}
830 \pm 85 \\
\text { AD } 1120
\end{array}
$$

$$
835 \pm 85
$$$$
\text { AD } 1115
$$

\section{Towosahgy State Archaeological site series}

Charcoal from fill of stockade trenches encircling center of Towosahgy State Archaeol site, 23Mi2, fortified ceremonial center for Mississippian tradition of SE Missouri, East Prairie, Missouri $\left(36^{\circ} 42^{\prime} \mathrm{N}\right.$, 
$89^{\circ} 14^{\prime} \mathrm{W}$ ). Coll by J C Cotter; subm 1972 by M D Southard, Towosahgy State Archaeol site.

N-1250. Towosaghy 1 (CS2-70)

$815 \pm 85$

From burned post in Stockade Trench A, Grid Unit 857R1353.

N-1251. Towosaghy 2 (CS1-71)

$1060 \pm 85$

From burned post in Stockade Trench A, Grid Unit $703 \mathrm{~N} / 130 \mathrm{E}$.

N-1252. Towosaghy 3 (CS2-71)

$930 \pm 95$

From base of Stockade Trench B, Grid Unit 694.8N.

\section{N-1253. Towosaghy 4 (CS3-71)}

From burned post in Stockade Trench A.

$$
\begin{array}{r}
1200 \\
\text { AD } 750
\end{array}
$$

General Comment (MDS): dates seem too early and do not represent true age of stockade feature assoc with Cairo Lowland phase of Middle Mississippian occupation of Towosahgy. Previous date for post from Stockade A yielded $675 \pm 70$ (UGA-244).

\section{Pot Shelter series}

Material from stratified site of Pot Shelter (23CR149), E-central Missouri $\left(38^{\circ} 6^{\prime} \mathrm{N}, 91^{\circ} 10^{\prime} \mathrm{W}\right)$. First 2 samples come from Woodland occupation; next 3 from Archaic occupation. Coll 1971 by F E Schneider; subm by $\mathrm{R}$ Krause, Univ Missouri-Columbia. Comment (RK): excavation will be reported in the 3rd Rept to US Natl Park Service on Archaeol Salvage in Proposed Meramec Park Reservoir.

\section{N-1169. Pot Shelter 1}

$1300 \pm 110$

Charcoal from concentrated area of ash and charcoal representing hearth, Feature 7, 46 to $56 \mathrm{~cm}$ below surface, sealed under a pile of large rocks.

\section{N-1170. Pot Shelter 2}

$4150 \pm 125$

$2200 \mathrm{BC}$

Charcoal from excavation Level 15,107 to $114 \mathrm{~cm}$ below surface, within both a cultural and soil transition zone between upper Woodland and lower Archaic deposits. Pottery first appears stratigraphically in Level 14.

\section{N-1171. Pot Shelter 3}

Charcoal from Level 23,160 to $175 \mathrm{~cm}$ below surface. Side-notched dart point was next to Feature 16, burned clay fire hearth. 
N-1172. Pot Shelter 4

$5600 \pm 125$

3650 BC

Charcoal from Level 26, 188 to $198 \mathrm{~cm}$ below surface, where burned clay fire hearths, Features 18, 19 and 20, first appear.

\section{N-1173. Pot Shelter 5}

$6480 \pm 145$

Charcoal combined from Level 30, 221 to $241 \mathrm{~cm}$ below surface and from burned clay fire hearth, Feature 24 .

\section{N-1174. Smith Shelter (23CR80)}

$805 \pm 100$

Charcoal from Feature 4, Sq 2, excavation Level 4 at Smith Shelter, E-central Missouri $\left(38^{\circ} 6^{\prime} \mathrm{N}, 91^{\circ} 10^{\prime} \mathrm{W}\right)$, in which main occupation is Late Middle Woodland. Feature consisted of circular area of charcoal, max diam $30 \mathrm{~cm}$, depth $5 \mathrm{~cm}$, as expected for a burned post. A rockerstamped sherd came from level below. Coll 1971 by F E Schneider; subm by $\mathrm{R}$ Krause.

\section{N-1175. Patton site (23CR60)}

$1010 \pm 100$

Charcoal from composite sample from Level 3 to 6,25 to $53 \mathrm{~cm}$ below surface, in large pit, Feature 3 in Patton site, E-central Missouri $\left(38^{\circ} 2^{\prime} \mathrm{N}, 91^{\circ} 14^{\prime} \mathrm{W}\right)$. The pit, $101 \times 99 \mathrm{~cm}$, contained cultural debris, charcoal, and burned limestone and was probably roasting or cooking pit. Coll 1971 by F E Schneider; subm by R Krause.

\section{Saba Shelter series}

Material from Saba Shelter (23BE149), Benton Co, Missouri $\left(38^{\circ}\right.$ $12^{\prime} \mathrm{N}, 93^{\circ} 28^{\prime} \mathrm{W}$ ). Site is stratified and to depth at least $183 \mathrm{~cm}$ below surface are Woodland materials: ceramics, abundant lithic artifacts and debitage, and floral and faunal material. Coll 1970 by R Vehik; subm by $\mathbf{R}$ Krause.

\section{N-1176. Saba Shelter 1}

$1400 \pm 100$

Charcoal from top of small pit, $30 \mathrm{~cm}$ below surface, containing lithic artifacts, debitage, worked bone, charcoal, seeds, nuts, burned and unburned bone, shell, and snails.

\section{N-1177. Saba Shelter 2}

$2070 \pm 100$

Charcoal from dark brown humus of Stratum 2, 61 to $91 \mathrm{~cm}$ below surface, assumed assoc with Woodland occupation, because of cordmarked and plain pottery, Scallorn-like points, Rice side-notched points, and other lithic artifacts.

\section{McRoberts Oneota site series}

Charcoal from McRoberts site (23SA5), Saline Co, Missouri $\left(39^{\circ} \mathrm{N}\right.$, $93^{\circ} \mathrm{W}$ ). Site consists of a group of small horticultural outposts occupied during late spring and late summer-early fall seasons for planting and 
harvesting crops in Missouri R flood plain. Coll and subm 1972 by R Krause.

N-1269. MeRoberts Oneota site 1 (CN 9)

N-1106. McRoberts Oneota site 2 (CN 10)

N-1270. McRoberts Oneota site 3 (CN 12)

N-1271. McRoberts Oneota site 4 (CN 13)

\section{N-1272. McRoberts Oneota site 5 (CN 16)}

N-1273. McRoberts Oneota site 6 (CN 16)

\author{
Modern \\ AD 1650 \\ $300 \pm 95$ \\ Modern \\ Modern \\ AD 1840 \\ $110 \pm 75$ \\ AD 1650 \\ $300 \pm 75$ \\ AD 1560
}

N-1274. McRoberts Oneota site 7 (CN 19)

General Comment (RK): because recovered trade items, eg, glass beads, brass kettle fragments, and lead rifle ball were found, site was expected to date between AD 1600 to 1800 . Dates of N-1106, -1272-1274 fall within or near expected age. N-1269 is equivalent to N-1106 in terms of archaeol context and assoc; both samples were from same prepared hearth. Date of N-1269 is, thus, unacceptable. N-1270 and -1271 were from a prepared hearth assoc with Oneota potsherds; these 2 dates are unacceptable also, but their consistency suggests an error in field interpretation.

\section{Santa Luisa series}

\section{Mexico}

Material from archaeol site $30 \mathrm{GZl}$ at Santa Luisa, Mexico $\left(20^{\circ} 28^{\prime}\right.$ $\left.\mathrm{N}, 97^{\circ} 4^{\prime} \mathrm{W}\right)$. Coll and subm 1970 by S J K Wilkerson. Comment $(\mathrm{SJKW})$ : dates help establish reliable chronology for $\mathrm{N}$-central Veracruz area, particularly for Formative periods.

\section{N-912. Santa Luisa 1}

Charcoal dispersed in earth from hearth, Trench 5, Level 14, depth 250 to $260 \mathrm{~cm}$. Estimated age: 600 to $400 \mathrm{BC}$.

\section{N.913. Santa Luisa 2}

$4740 \pm 100$

Dispersed charcoal from Trench 5, Level 25, depth 460 to $480 \mathrm{~cm}$. Assoc with obsidian flakes and oyster shells in deepest level. Estimated age: 600 to $1000 \mathrm{BC}$.

N-914. Santa Luisa 3

$2370 \pm 105$

Charcoal from Trench 5, Level 11, depth 190 to $210 \mathrm{~cm}$. Estimated age: 500 to $200 \mathrm{Bc}$. 
N-915. Santa Luisa 4
Total organic carbon in $900 \mathrm{~g}$ of ash from interior of Structure

$2280 \pm 120$ A-sub 4, earliest ceremonial architecture found at site. Trench 3-B, depth 270 to $272 \mathrm{~cm}$. Estimated age: AD 300 to 600 .

N-916. Santa Luisa 5

$2730 \pm 105$

780 вC

Charcoal from Trench 5, Level 13, depth 230 to $250 \mathrm{~cm}$. Estimated age: 600 to 400 BC.

\section{N-917. Santa Luisa 6}

$1110 \pm 100$

Charcoal from firepit, Trench 2, Level 6, depth 110 to $120 \mathrm{~cm}$. Estimated age: AD 600 to 900 .

\section{N-918. Santa Luisa 7}

$2710 \pm 105$

Charcoal from Trench 5, Level 12, depth 210 to $230 \mathrm{~cm}$. Estimated age: 500 to $200 \mathrm{BC}$.

\section{N-919. Santa Luisa 8}

$1600 \pm 100$

Charcoal from Trench 3-C, depth 415 to $425 \mathrm{~cm}$. Estimated age: AD 400 to 700 .

N-920. Santa Luisa 9

$4410 \pm 130$

Inorganic carbon from carbonaceous ash, Trench 2, Level 9 and 10 , depth 178 to $183 \mathrm{~cm}$. Estimated age 300 to 0 BC.

\section{Nexpa series}

Charcoal from archaeol remains at Nexpa, Morelos, Mexico $\left(18^{\circ}\right.$ $\left.31^{\prime} \mathrm{N}, 99^{\circ} 9^{\prime} \mathrm{W}\right)$. Coll and subm 1970 by $\mathrm{D} \mathrm{C}$ Grove, Univ Illinois at Urbana-Champaign.

\section{N-941. Nexpa 1}

$3100 \pm 120$

1150 BC

From Pit Na-1A, N sidewall, assoc with walls of apparent Early Formative age, $105 \mathrm{~cm}$ below ground surface.

N.942. Nexpa 2

$3100 \pm 120$

$1150 \mathrm{BC}$

From Pit Na-1, assoc with wall and apparent house floor of Early Formative age, 125 to $140 \mathrm{~cm}$ below ground surface.

N-943. Nexpa 3

$3170 \pm 120$

$1220 \mathrm{BC}$

From Pit Na-3, from packed clay house floor of apparent Early Formative age, 65 to $75 \mathrm{~cm}$ below ground surface. 
N-944. Nexpa 4

From Pit Na-4, from ash layer adjacent to Burial 1 containing Tlatilco-Rio Cuautla style burial offerings. Age: Late Early Formative.

N-945. Nexpa 5

$2930 \pm 130$

From Pit Nc-2, Layer VI. Age: Early Formative.

980 в

N.946. Nexpa 6

$3010 \pm 120$

From Pit Nc-2, Level VII. Age: Early Formative.

$1060 \mathrm{BC}$

\section{Chalcatzingo series}

Charcoal from archaeol site at Chalcatzingo, Morelos, Mexico $\left(18^{\circ}\right.$ $\left.41^{\prime} \mathrm{N}, 99^{\circ} 46^{\prime} \mathrm{W}\right)$. Coll and subm 1972 by D C Grove.

\section{N-1402. Chalcatzingo 1}

$2620 \pm 80$

From excavation Unit 112-114S, 0-2E, at depth $57 \mathrm{~cm}$. Cemetery area on central plaza. Age: Middle Formative.

\section{N-1403. Chalcatzingo 2}

$2480 \pm 80$

From Unit 112-114S, 2-4E, at depth 20 to $42 \mathrm{~cm}$. Cemetery area on central plaza. Age: Middle Formative.

\section{N-1404. Chalcatzingo 3}

From Unit 114-116S, 0-2E, at depth 40 to $60 \mathrm{~cm}$, near burial offering No. 94 in cemetery area on central plaza. Age: Middle Formative.

N-1405. Chalcatzingo 4

$2700 \pm 95$

$750 \mathrm{BC}$

From Unit 114-116S, 2-4E, at depth 40 to $60 \mathrm{~cm}$. Cemetery area on plaza. Age: Middle Formative.

\section{N-1406. Chalcatzingo 5}

$2890 \pm 100$

From Unit 118-120S, 0-2E, at depth $90 \mathrm{~cm}$. Cemetery area on central plaza. Age: Middle Formative.

\section{N-1407. Chalcatzingo 6}

From Trench $90-87$, at depth 360 to $380 \mathrm{~cm}$ of central plaza. Age: Middle Formative.

N-1408. Chalcatzingo 7

From Trench $84-80$, at depth 180 to $220 \mathrm{~cm}$, central plaza. Age: Middle Formative. 
N-1409. Chalcatzingo 8

$3010 \pm 95$

$1060 \mathrm{BC}$

From Trench $75-71$ at depth 370 to $390 \mathrm{~cm}$, central plaza. Age: Middle Formative.

\section{N-1410. Chalcatzingo 9}

$2620 \pm 90$

From Trench $60-63.5$ at depth $233 \mathrm{~cm}$, central plaza. Assoc with bone and architectural features. Age: Middle Formative.

\section{N-1411. Chalcatzingo 10}

$$
2840 \pm 95
$$

890 BC

From excavation Area 110-112S 16-18E, at depth 40 to $80 \mathrm{~cm}$, central plaza. Assoc with architectural features. Age: Middle Formative.

\section{N-1412. Chalcatzingo 11}

$2910 \pm 130$

From Area 110-112S, 16-18E, at depth 190 to $210 \mathrm{~cm}$, central plaza. Assoc with architectural features. Age: Middle Formative.

\section{N-1413. Chalcatzingo 12}

$3320 \pm 80$

1370 BC

From Area 14-17.5S, 39-40E, at depth 180 to $220 \mathrm{~cm}$, E edge of long platform mound bounding $\mathrm{N}$ side of central plaza. Age: probably Early Formative.

\section{N-1414. Chalcatzingo 13}

$1390 \pm 75$

From Unit $0-2 \mathrm{~S}, 0-2 \mathrm{E}$, at depth 20 to $40 \mathrm{~cm}$, from terrace of Middle Formative and Classic house structures. Age: Classic.

\section{N-1415. Chalcatzingo 14}

$1350 \pm 75$

From Unit 4-6S, $0-2 \mathrm{~W}$, at depth 31 to $40 \mathrm{~cm}$, Soil Zone B, from terrace of Middle Formative and Classic house structures. Age: Classic.

\section{N-1416. Chalcatzingo 15}

$3030 \pm 130$

From Unit $8-10 \mathrm{~S}, 0-2 \mathrm{~W}$, at depth 140 to $160 \mathrm{~cm}$, Soil Zone D, from terrace of Middle Formative house structure.

\section{N-1417. Chalcatzingo 16}

From Unit $8-10 \mathrm{~S}, 2-4 \mathrm{~W}$, at depth 60 to $80 \mathrm{~cm}$, Soil Zone B, from terrace of Middle Formative house structure.

\section{Craig Phadrig series}

\section{Great Britain}

Material from archaeol remains at Craig Phadrig, Inverness, $\mathrm{N}$ Scotland $\left(57^{\circ} 29^{\prime} \mathrm{N}, 4^{\circ} 14^{\prime} \mathrm{W}\right)$. Coll and subm 1971 by Alan Small, Univ Dundee. 
N-1118. Craig Phadrig 1 (CP25)

Charred timber between layers of upper and lower occupations overlying collapse of rampart.

N-1119. Craig Phadrig 2 (CP107)

$1540 \pm 85$

Charcoal from upper occupation layer.

AD 410

N-1120. Craig Phadrig 3 (CP114)

$2250 \pm 100$

Dispersed charcoal from wooden beam in earthen rampart.

N-1122. Craig Phadrig 4 (CP128)

$2280 \pm 100$

330 BC

Charcoal from base of wall buried in rubble, $3.5 \mathrm{~m}$ high.

N-1123. Craig Phadrig 5 (CP130)

$2220 \pm 100$

Charcoal under buried wall.

270 вс

\section{N-1124. Craig Phadrig 6 (CP133)}

$2320 \pm 105$

$\mathbf{3 7 0}$ вC

Charcoal and peat from face of buried wall, $50 \mathrm{~cm}$ below top of wall.

\section{N-1238. Reswallie Farm}

$3160 \pm 70$

Human bone from Reswallie Farm, Rescobie, Scotland $\left(56^{\circ} 40^{\prime} \mathrm{N}\right.$, $2^{\circ} 49^{\prime} \mathrm{W}$ ). Inhumation in short cist. Grave goods included food vessel and flint flake. Coll 1967 and subm by H Coutts, Dundee Mus. Comment: bone collagen dated.

\section{N-1239. Cookston Farm}

$3550 \pm 85$

$1600 \mathrm{BC}$

Human bone from Cookston Farm, Eassie, Scotland $\left(56^{\circ} 37^{\prime} \mathrm{N}\right.$, $\left.3^{\circ} 4^{\prime} \mathrm{W}\right)$. Inhumation in short cist. Grave goods included beaker and bone button. Coll 1970 and subm by $\mathrm{H}$ Coutts. Comment: bone collagen dated.

N-1240. Glamis

$3390 \pm 90$

$1440 \mathrm{BC}$

Human bone from Glamis, Angus, Scotland (56 $\left.36^{\circ} \mathrm{N}, 3^{\circ} \mathrm{W}\right)$. Inhumation in short cist. Grave goods included food vessel sherd. Coll 1947 by D R Dow; subm by H Coutts. Comment: bone collagen dated.

\section{Green Cairn series}

Material from archaeol remains at Green Cairn, Fife, Scotland $\left(56^{\circ} 5^{\prime} \mathrm{N}, 3^{\circ} 35^{\prime} \mathrm{W}\right)$. Coll and subm 1972 by L M Wedderburn, Dundee City Mus. Estimated age: 1000 to $3500 \mathrm{BP}$. 
N.1318. Green Cairn 1 (G.C./T1/4/S1)

$2130 \pm 100$

Charcoal from twigs.

$180 \mathrm{BC}$

N-1375. Green Cairn 2 (G.C./T5/5/S2)

$2340 \pm 95$

Carbon rich material from post hole.

N-1376. Green Cairn 3 (G.C./T1/4/S3)

$2490 \pm 90$

Charcoal from burned timber beam.

540 BC

General Comment (LMMW): dates represent construction, destruction, and occupation of defended settlement of Scottish Iron age and are supported by stratigraphy of excavated areas.

\section{Chondwe series}

\section{E. Africa}

Charcoal from Early Iron age site at Chondwe, Copperbelt Prov, W Zambia (13 $\left.12^{\prime} \mathrm{S}, 28^{\circ} 47^{\prime} \mathrm{E}\right)$. Coll by $\mathrm{N}$ Filmer and E Mills, Ndola, Zambia; subm 1970 by B M Fagan, Univ California, Santa Barbara. Comment (BMF): may date beginnings of occupation. Probable date: ca 1100 BP.

N.997. Chondwe 1

From Trench 2, Sq 5, depth 1.98 to $2.06 \mathrm{~m}$.

N-998. Chondwe 2

From Trench 2, Sq 5, depth 2.06 to $2.13 \mathrm{~m}$.
$1150 \pm 145$ AD 800

$1440 \pm 160$ AD 510

\section{Kansanshi series}

Material from Kansanshi copper mine, Zambia $\left(11^{\circ} 40^{\prime} \mathrm{S}, 26^{\circ} 30^{\prime} \mathrm{E}\right)$. Coll by M S Bisson; subm 1972 by B M Fagan.

N-1281. Kansanshi 1 $360 \pm 80$

Charcoal from distinct hearth at contact of orange-gray rubble and sandy orange layer at depth $3.20 \mathrm{~m}$ in fill, Trench $\mathrm{I}, 4.2$ to $4.65 \mathrm{~m} \mathrm{~S}$ of datum.

N-1282. Kansanshi 2

$295 \pm 80$

Charcoal from angular rubble and brown sandy matrix at depth $1.73 \mathrm{~m}$ in fill, Trench $\mathrm{I}, 5.42 \mathrm{~m} \mathrm{~S}$ of datum.

N.1283. Kansanshi 3 $1320 \pm 85$

Charcoal from daga pit in probably earliest village horizon at Kansanshi, at depth 45 to $75 \mathrm{~cm}$, Pit II, Site Ksm. Assoc with copper working. 
N-1284. Kansanshi 4

$$
1190 \pm 85
$$

AD 760

Charcoal from interface between black and yellow layer at depth $37 \mathrm{~cm}$, Site Ksm. Assoc with early type pottery.

\section{N-1285. Kansanshi 5}

Charcoal from orange clay at base of layer, depth $26 \mathrm{~cm}$, containing earlier type of pottery and underlain by (?) Middle Stone age tools, Site Ksm.

\section{N-1286. Kansanshi 6}

$$
1550 \pm 90
$$

\section{AD 400}

Charcoal from Pit I fill at depth $47 \mathrm{~cm}$. Assoc with Late Iron age pottery and anthill furnace fragments, Site Ksm.

General Comment (MSB): N-1281 and -1282 were from rubble backfill of ancient copper mine at Kansanshi hill. They date final period of great prehistoric activity, obliterating all traces of earlier copper mining. $\mathrm{N}-1283$ to -1286 were all from prehistoric smelting area adjacent to Kansanshi mine. N-1283 dates lst phase of Early Iron age activity at the mine while $\mathrm{N}-1284$ dates 2nd phase. Both phases are characterized by distinct ceramic assemblages. Dates agree fully with already known Early Iron age dates from NW Zambia. Date of N-1285 was earlier than expected and probably belongs to underlying layer below Iron age pottery. Date of N-1286 was earlier than expected. Subsequent study of pottery from this pit shows that it falls within range of variation of lst phase of Early Iron age.

\section{Chundu series}

Charcoal from Chundu site, Livingstone Dist, S Prov, Zambia $\left(17^{\circ}\right.$ $\left.35^{\prime} \mathrm{S}, 25^{\circ} 41^{\prime} \mathrm{E}\right)$. Coll 1970 and subm by J O Vogel, Livingstone Mus.

\section{N-1137. Chundu 1 (Zlm-32)}

$1190 \pm 100$

Charcoal from depth $1.2 \mathrm{~m}$ in ashpit, Trench 5 .

\section{N-1138. Chundu 2 (Zlm-33)}

Charcoal from depth $1.2 \mathrm{~m}$ in ashpit, Trench 2.

$$
\begin{array}{r}
1290 \\
\text { AD } 660
\end{array}
$$

\section{N-1139. Chundu 3 (Zlm-34)}

$$
1160 \pm 160
$$

AD 790

Charcoal from depth $0.6 \mathrm{~m}$ within horizon containing village-assoc cultural material, Trench 4. Comment (JOV): earlier sample N-668 $(220 \pm 170: \mathrm{R}, 1970, \mathrm{v} 12, \mathrm{p} 572)$ was inconsistent with typologic evidence.

\section{Zambesi series}

Charcoal from Zambesi site, Livingstone Dist, S Prov, Zambia ( $17^{\circ}$ $49^{\prime} \mathrm{S}, 25^{\circ} 37^{\prime} \mathrm{E}$ ), from provenance believed assoc with Early Iron age horizon. Coll 1971 and subm by J O Vogel. 
N-1140. Zambesi 1 (Zlm-35)

$1410 \pm 130$

Charcoal from large pit assoc with hut and filled with Early Iron age pottery, Trench 10 .

N-1141. Zambesi 2 (Zlm-36)

Charcoal from large pit in Trench 7 .

N-1142. Zambesi 3 (Zlm-37)

Charcoal from ashpit at $0.8 \mathrm{~m}$ in Trench 6 .

N-1143. Zambesi 4 (Zlm-38)

Charcoal assoc with hut daga in Trench 8.

N-1144. Zambesi 5 (Zlm-39)

Charcoal assoc with hut in Trench 10.

N-909. Turkwel R Irrigation scheme, Site U

Charcoal from possible hearth remnant exposed at surface of winddeflated site, near Lorogumu, Turkana Dist, Kenya $\left(2^{\circ} \mathrm{N}, 36^{\circ} \mathrm{E}\right)$. Late Stone age tools and incised pottery were adjacent to charcoal. Coll and subm 1970 by L H Robbins, Michigan State Univ.

\section{Lothagam Hill series}

Material from archaeol sites near Lothagam Hill, S Turkana Dist, Kenya $\left(2^{\circ} \mathrm{N}, 36^{\circ} \mathrm{E}\right)$. Subm 1971 by L H Robbins.

N-1100. Lothagam Hill (ZU-4)

$8420 \pm 170$

$6470 \mathrm{BC}$

Shell from compact grayish sand level, 20 to $30 \mathrm{~cm}$ below surface, assoc with cultural material including Late Stone age flaking debris and pottery. Coll 1969 by L H Robbins. Comment (LHR): shell from Site ZU-6, ca 100m apart from Site ZU-4 yielded $7960 \pm 140$ (N-813: R, 1972, v 14, p 237).

\section{N-1101. Lothagam Hill 2 (ZU-5)}

$6010 \pm 160$

Shell from exposure of shell beds, 35 to $40 \mathrm{~cm}$ below surface, Site $\mathrm{ZU}-5,<1.6 \mathrm{~km}$ from ZU-4. Late Stone age flaking debris and pottery found at adjacent surface. Coll 1969 by L H Robbins and J I Ebert.

\section{N-1102. Lothagam Hill 3a (BB-9)}

$8230 \pm 180$ $6280 \mathrm{BC}$

Shell from dark organic lake sediment at Site BB-9, ca $0.4 \mathrm{~km}$ from $S$ end of Lothagam Hill, yielding human skeletal remains, Late Stone age artifacts, bone points, and fauna. Coll 1969 by L H Robbins. 
N-1103. Lothagam Hill $3 \mathrm{~b}$

Black organic sediment from above site, 2 to $16 \mathrm{~cm}$ below surface. Comment (LHR): material did not serve as independent check against N-1102.

\section{N-1076. Lukenya Hill}

$1330 \pm 100$

Charcoal from prehistoric rockshelter, Site GvJm/22, Lukenya Hill, Machakos Dist, Kenya $\left(1^{\circ} 29^{\prime} \mathrm{S}, 37^{\circ} 4^{\prime} \mathrm{E}\right)$, from hearth $50 \mathrm{~cm}$ below surface. Coll and subm 1971 by R M Gramly, Univ Nairobi. Comment (RMG): should date 2 pottery wares and provide upper limit for Narosura ware.

\section{N-1066. Manda}

$$
\begin{array}{r}
1240 \pm 100 \\
\text { AD } 710
\end{array}
$$

Water-logged wood, probably mangrove, from one of a series of large piles ca $20 \mathrm{~cm}$ diam, sunk into mud $3.5 \mathrm{~m}$ thick filling what must have been open creek at Manda, Lamu Dist, Kenya $\left(2^{\circ} 14^{\prime} \mathrm{S}, 40^{\circ} 58^{\prime} \mathrm{E}\right)$. Present shoreline is ca $15 \mathrm{~m}$ away. Mud surface is at approx present midtide level and remains permanently water-logged with salt water. Coll 1970 by H N Chittick; subm by R C Soper, British Inst E Africa. Comment (HNC): sample can be assigned to early stage of city of Manda and probably belongs to 9 th or 10 th century $\mathrm{AD}$, based on pottery imported from Persian Gulf.

\section{New Seronera Game Lodge series}

Charcoal from cave site at New Seronera Game Lodge, Serengeti Natl Park, Tanzania $\left(2^{\circ} 25^{\prime} \mathrm{S}, 34^{\circ} 50^{\prime} \mathrm{E}\right)$. Coll 1971 by J R F Bower; subm by R C Soper.

\section{N-1067. New Seronera Game Lodge 1}

$2020 \pm 115$

Charcoal from depth 30 to $40 \mathrm{~cm}$ from surface, Sq B-2, Site SE-3, underlying stratigraphic break in cave deposits formed by boulder rubble. Assoc with pottery of possible East African "Neolithic" affinity and microlithic industry, mostly obsidian. Comment (JRFB): consistent with range of dates for pottery of $\mathrm{E}$ African "Neolithic" affinity and provides reliable terminal date for pottery (Gumban A) recovered below rubble.

\section{N-1068. New Seronera Game Lodge 2}

$265 \pm 100$

Charcoal from depth 10 to $20 \mathrm{~cm}$ below surface, Sqs B-1 and C-1, Site SE-3, overlying stratigraphic break in cave deposits. Assoc with Iron age pottery (twisted cord rouletting and various forms of incised decoration) and microlithic industry, mostly quartz. Comment (JRFB): unexpected younger age due to thorough disturbance of deposits overlying boulder rubble. 
N-1158. New Seronera Game Lodge 3

AD 1670

Charcoal recovered at depth 20 to $30 \mathrm{~cm}$ from surface in Sq C-10, Site SE-4, in colluvium on rock terrace a few meters downslope from cave (SE-3), assoc with thin-walled (av 5mm) pottery with incised, panelled decoration and microlithic industry of mostly quartz. Comment (JRFB): date considerably younger than expected. Contamination must have been great, since no natural stratigraphy was observed and immediately overlying layer contained very recent pottery.

\section{Kisii series}

Material from various sites in Kisii Dist, W Kenya. Coll 1971 and subm by J R F Bower, Lake Forest College.

\section{N-1234. Kisii 1 (Gs Jd 6)}

$2090 \pm 170$

Charcoal from depth ca $55 \mathrm{~cm}$ in reddish, clayey colluvium $\left(0^{\circ} 39^{\prime} \mathrm{S}\right.$, $34^{\circ} 49^{\prime} \mathrm{E}$ ), assoc with pottery provisionally labelled Kisii Soft Ware.

\section{N-1235. Kisii 2 (Gs Jd 21)}

$1190 \pm 75$

Charcoal from depth ca $70 \mathrm{~cm}$ in brown, loamy colluvium $\left(0^{\circ} 40^{\prime} \mathrm{S}\right.$, $34^{\circ} 55^{\prime} \mathrm{E}$ ), assoc with Kisii Soft Ware on burnt clay floor with hearth stones.

\section{N-1236. Kisii 3 (Gt Je 7)}

AD 1785

$165 \pm 90$

Charcoal from depth ca $60 \mathrm{~cm}$ in midden-stained soil $\left(0^{\circ} 49^{\prime} \mathrm{S}\right.$, $\left.34^{\circ} 44^{\prime} \mathrm{E}\right)$, assoc with pottery provisionally labelled Button-Necked, lying among hearth stones (?).

\section{N-1237. Kisii 4 (Gt Je 9)}

$1650 \pm 90$

Charcoal from depth ca $60 \mathrm{~cm}$ in reddish brown colluvium $\left(0^{\circ} 53^{\prime} \mathrm{S}\right.$, $34^{\circ} 43^{\prime} \mathrm{E}$ ), assoc with pottery of Indeterminate type (decoration consisted in short vertical incisions around rim, horizontal bands of punctuations around body, and multiple parallel U-grooved incisions), chipped stone tools, and very friable bone.

General Comment (JRFB): wide gaps in dates are not surprising, since KSW ware (N-1234 and -1235) differs markedly from both BNP ware (N-1236) and Indeterminate ware (N-1237), and the latter 2 are equally divergent in appearance. Of interest, however, is the gap between the 2 KSW dates and the fact that they bracket date for Indeterminate ware.

\section{Ngungani series}

Charcoal from archaeol site at Ngungani, Chyulu Hills, Machakos Dist, Kenya $\left(2^{\circ} 35^{\prime} \mathrm{S}, 37^{\circ} 50^{\prime} \mathrm{E}\right)$. Coll and subm 1972 by $\mathrm{R}$ C Soper. Comment (RCS): expected age is within present millennium and comparable to N-290 (435 $\pm 105:$ R, 1968, v 10, p 342). 
N-1316. Ngungani 1

NG72, Hpl4 WI (4). From depth $90 \mathrm{~cm}$, assoc with hut floor.

\section{N-1317. Ngungani 2}

$430 \pm 75$

NG72, HcJp3 (3). From depth $50 \mathrm{~cm}$ in large ash heap.

General Comment (RCS): N-1317 compares closely with N-290. N-1316 was expected to be contemporary with other 2 on preliminary examination of pottery, but a detailed study has not yet been made; sample came from very localized area and may have been contained in pre-existing animal burrow.

\section{Kwelikwiji series}

Material from Kwelikwiji site, Ngulu Hills, Morogoro Area, Tanzania $\left(6^{\circ} 6^{\prime} \mathrm{S}, 37^{\circ} 33^{\prime} \mathrm{E}\right)$, assoc with Early Iron age pottery of Kwale type. Coll and subm 1972 by R C Soper.

\section{N-1287. Kwelikwiji 1}

$3210 \pm 85$

Charcoal from depth 18 to $22 \mathrm{~cm}$ in red-brown clay.

\section{N-1288. Kwelikwiji 2}

$3050 \pm 85$

Charcoal from depth 30 to $40 \mathrm{~cm}$, in red-brown clay.

1100 BC

General Comment (RCS): dates are $>1000 \mathrm{yr}$ earlier than comparable sites to N. Kwale ware sherds were from 15 to $45 \mathrm{~cm}$ below surface, and nondescript quartz industry from 35 to $60 \mathrm{~cm}$, with abundant charcoal also from 15 to $45 \mathrm{~cm}$. Either the charcoal dates the stone industry and there has been some disturbance, or the charcoal comes from a very old tree (unlikely to be this old), or Kwale ware really is this old and dates back to pre-Iron age.

\section{N-1145. Nhunguza Ruin}

$370 \pm 100$

Piece of structural timber supporting roof of main hut in Zimbabwetype ruin from Nhunguza ruin (Garlake, 1973a), S Rhodesia (17 $23^{\prime} \mathrm{S}$, $31^{\circ} 14^{\prime} \mathrm{E}$ ). Coll and subm 1971 by P S Garlake, Univ of Ife.

\section{Ruanga Ruin series}

Charcoal from Ruanga ruin (Garlake, 1973a), S Rhodesia (17 2' S, $\left.31^{\circ} 41^{\prime} \mathrm{E}\right)$. Coll and subm 1971 by P S Garlake.

N-1146. Ruanga ruin 1

$\mathbf{4 5 0} \pm \mathbf{8 5}$

AD 1500

Charcoal from depth $45 \mathrm{~cm}$ in midden in Zimbabwe-type ruin, assoc with "Zimbabwe-type" pottery and walling. 
N-1147. Ruanga ruin 2

$775 \pm 100$

Charcoal from depth 1.1 to $1.3 \mathrm{~m}$ in midden, assoc with "Musengezitype" pottery, underlying "Zimbabwe" deposits.

N-1148. Tafuna Hill

$1070 \pm 105$ AD 880

Charcoal from Tafuna Hill, S Rhodesia $\left(17^{\circ} 23^{\prime} \mathrm{S}, 31^{\circ} 32^{\prime} \mathrm{E}\right)$, from depth 20 to $30 \mathrm{~cm}$ in occupation level of Early Iron age Chitope-ware settlement (Garlake, 1971). Coll and subm 1971 by P S Garlake.

Obalara's Land series

Charcoal from site, Obalara's Land, Ife town, W Nigeria $\left(7^{\circ} 29^{\prime} \mathrm{N}\right.$, $\left.4^{\circ} 32^{\prime} \mathrm{W}\right)$. Assoc with apparent shrine containing terracotta sculptures of "Classical" period. Estimated age ca 12th to 14th centuries AD. Coll and subm 1972 by P S Garlake (Garlake, 1973b).

N-1390. Obalara's Land 1

$480 \pm 95$

Charcoal from gravel surrounding concentration of pottery probably representing shrine offering.

N-1391. Obalara's Land 2

$580 \pm 60$

Charcoal from gravel overlying a group of terracotta sculptures.

N-1392. Obalara's Land 3

$760 \pm 85$

Charcoal from gravel underlying further concentration of pottery probably representing shrine offering.

N-1393. Obalara's Land 4

$625 \pm 75$

AD 1325

Charcoal from gravel amongst concentration of human bones close to group of terracotta sculptures.

\section{Begho series}

Charcoal from archaeol remains at Begho, trading town in AD 1400 to 1700 , near Hani, Brong Ahafo Region, Ghana $\left(7^{\circ} 15^{\prime} \mathrm{N}, 2^{\circ} 28^{\prime} \mathrm{E}\right)$. Coll and subm 1970 by M Posnansky, Univ Ghana (Posnansky, 1971; Wilks, 1961).

N-929. Begho 1

$$
240 \pm 100
$$

From Pit I, Layer 6, 3rd occupation layer assoc with many sherds. $1.24 \mathrm{~m}$ below ground surface.

N.930. Begho 2

From Pit M33, surface of Layer 6, assoc with lowest pottery horizon within very compact orange subsoil. $1.1 \mathrm{~m}$ below ground surface. 
N-931. Begho 3

$285 \pm 100$

From Pit K39, Layer 4, midden deposit below floor of 17 th century house. Assoc with mass of pottery. $0.8 \mathrm{~m}$ below ground surface.

\section{N-932. Begho 4}

$500 \pm 100$

From Pit K39, Layer 7, underlying floor of building dated by assoc small finds to latter half of 17 th century. Assoc with well preserved burial in shallow pit. $1.3 \mathrm{~m}$ below ground surface.

\section{N-1430. Begho 5}

$410 \pm 75$

From Pit I, Layer 15, assoc with pottery and bones. $3.2 \mathrm{~m}$ below ground surface.

\section{Coronation Park series}

Charcoal from Coronation Park, Salisbury, Rhodesia $\left(17^{\circ} 50^{\prime} \mathrm{S}\right.$, $31^{\circ} 6^{\prime} \mathrm{E}$ ). Coll and subm 1971 by $\mathrm{T} \mathrm{N}$ Huffman, Natl Mus and Monuments, Rhodesia.

\section{N-978. Coronation Park 1}

$1240 \pm 100$

From sealed pit belonging to Coronation facies of Gokomere tradition (Huffman, 1971a).

\section{N-979. Coronation Park 2}

$970 \pm 100$

From village level belonging to Maxton facies of Gokomere tradition (Garlake, 1967), stratified above N-978. Comment (TNH): 1st dates for Coronation and Maxton facies, and they demonstrate a $1000 \mathrm{yr}$ continuum of Gokomere tradition in Mashonaland.

\section{N-1275. Makuru}

$1260 \pm 65$ AD 690

Charcoal from Early Iron age Zhizo site at Makuru (Huffman, 1973), $16 \mathrm{~km} \mathrm{~W}$ of Shabani, Rhodesia $\left(20^{\circ} 19^{\prime} \mathrm{S}, 29^{\circ} 58^{\prime} \mathrm{E}\right)$. Coll and subm by $\mathrm{T}$ N Huffman. Comment (TNH): agrees with dates from Zhizo level at Leopard's Kopje Main Kraal, SR-225 and I-4862 (Huffman, 1971b), and shows that Zhizo and Coronation are contemporary facies of 2nd phase of Gokomere tradition.

\section{REFERENCES}

Ariake Bay Research Group, 1965, Quaternary system of the Ariake and the Shiranui Bay Areas, with special reference to the Ariake soft clay: Assoc Geol Collaboration in Japan, mono 11, p 68.

Asaki, S, 1957, The cinerary urn with animal legs owned by the Tokuzo-ji Monastery: Archaeol Soc Nippon, Jour, v 43, p 54-58.

Furuuchi, S, 1971, Sanrizuka, an archaeological survey of site of New Tokyo International Airport: Hokuso Kosha, Chiba Pref. 
Garlake, P S, 1967, Excavations at Maxton Farm, near Shamva Hill, Rhodesia: Arnoldia Rhodesia, v 3, no. 9. Archaeol Bull, v 26, p 104.

1973a, Excavations at the Nhungoza and Ruanga ruins in Northern Mashonaland: S African Archaeol Bull, v 27, p 107-143.

1973b, Excavations at Obalara's Land, Ife; an interim report: W African Jour Archaeol, v 4.

Huffman, T N, 1971a, A guide to the Iron age of Mashonaland: Natl Mus Rhodesia, Occ Paper v 4, no. la, p 20-44.

1971b, Excavations at Leopard's Kopje Main Kraal; a preliminary report: S African Archaeol Bull, v 26, nos 101-102, p 85-89. no. 39 .

Kanto Regional Construction Bureau, Ministry of Construction, 1973, Report on the marine geotechnical survey at the central part of Tokyo Bay. Matthews, B, 1970, Age and origin of Aeolian sand in the Vale of York: Nature,
v 227, p 1234-1236.

1971, Soils in Yorkshire: Soil Survey Rec, no. 6, Soil Survey, Great Britain. Moriyama, A and Ozawa, M, 1972, Geomorphology and subsurface geology of the alluvial plain of the lower Yahagi River, Central Japan: Quaternary Research,
v 11, p 193-207.

Posnansky, M, 1971, Ghana and the origins of West African trade: African Quart. Wilks, I, 1961, The Northern factor in Ashanti history; Begho and the Manda:
African History, Jour, v 2, p 25. 